\title{
Tiotropium Bromide Has a More Potent Effect Than Corticosteroid in the Acute Neutrophilic Asthma Mouse Model
}

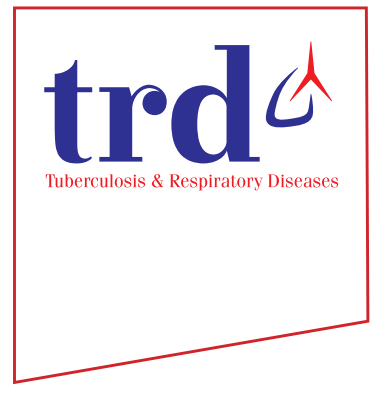

Tai Joon An, M.D. ${ }^{(1)}$, Ji Hye Kim, B.S., Chan Kwon Park, M.D. and Hyoung Kyu Yoon, M.D., Ph.D. Division of Pulmonary and Critical Care Medicine, Department of Internal Medicine, Yeouido St. Mary's Hospital, College of Medicine, The Catholic University of Korea, Seoul, Republic of Korea

Background: Neutrophilic asthma (NeuA) is usually resistant to corticosteroids. Tiotropium bromide (TIO) is a bronchodilator that is used as an add-on therapy to inhaled corticosteroid and long-acting $\beta 2$ agonist in asthma treatment. However, the role of TIO in NeuA is not fully known. Thus, the aim of this study was to evaluate the effect of TIO on NeuA compared to that of corticosteroids.

Methods: C57BL/6 female mice were sensitized with ovalbumin and lipopolysaccharide to induce neutrophilic inflammation. Dexamethasone (DEX) was administered on days 14, 17, 20, and 23. TIO was inhaled on days 21, 21, and 23. On day 24, mice were sacrificed. Airway hyper-responsiveness, levels of cytokines in bronchoalveolar lavage (BAL) and lung homogenates, and lung tissue histopathology were compared between the two groups.

Results: Neutrophil counts, $\mathrm{T}$ helper 2 cells $\left(\mathrm{T}_{\mathrm{H}} 2\right) / \mathrm{T}_{\mathrm{H}} 17$ cytokines, and pro-inflammatory cytokine in BAL fluids were elevated in the NeuA group. TIO group showed lower total cells, neutrophil counts, and eosinophil counts in BAL fluids than the DEX group $(\mathrm{p}<0.001, \mathrm{p}<0.05$, and $\mathrm{p}<0.001$, respectively). Airway resistance was attenuated in the TIO group but elevated in the NeuA group ( $\mathrm{p}<0.001$ ). Total protein, interleukin (IL)-5, and IL-17A levels in BAL fluids were lower in the TIO group than in the NeuA group (all $\mathrm{p}<0.05$ ).

Conclusion: TIO showed more potent effects than DEX in improving airway inflammation and attenuating airway resistance in NeuA.

Keywords: Neutrophilic Asthma; Tiotropium Bromide; Corticosteroid

\section{Introduction}

Address for correspondence: Hyoung Kyu Yoon, M.D., Ph.D.

Division of Pulmonary and Critical Care Medicine, Department of Internal Medicine, Yeouido St. Mary's Hospital, College of Medicine, The Catholic University of Korea, 63 ro 10, Yeongdeungpo-gu, Seoul 07345, Republic of Korea

Phone: 82-2-3779-2213, Fax: 82-2-780-3132

E-mail: cmcyhg@gmail.com

Received: Jul. 29, 2021

Revised: Sep. 29, 2021

Accepted: Oct. 30, 2021

Published online: Nov. 2, 2021

(c) It is identical to the Creative Commons Attribution Non-Commercial License (http://creativecommons.org/licenses/by-nc/4.0/). The Korean Academy of Tuberculosis and Respiratory Diseases.
Asthma is characterized by a chronic airway inflammation with heterogeneous features ${ }^{1,2}$. Severe asthma accounts for $5 \%-15 \%$ of all asthma cases. Impacts of severe asthma on the quality of life of patients, socioeconomic burden, and healthcare-related burden are enormous ${ }^{3,4}$. Its pathophysiology varies, including complex airway inflammation, non-eosinophilic inflammation, severe airway remodeling, and fixed airflow limitations. One of the proposed mechanisms of severe asthma is the increased neutrophilic inflammation of the airway, which is the main process of neutrophilic asthma (NeuA).

NeuA is a major category of non-eosinophilic asthma. It shows a low type 2 immune response and a non-atopic phenotype $^{6}$. Usually, NeuA is corticosteroid insensitive ${ }^{7,8}$. The T helper $1\left(\mathrm{~T}_{\mathrm{H}} 1\right)$ and $\mathrm{T}$ helper $17\left(\mathrm{~T}_{\mathrm{H}} 17\right)$ processes of NeuA are considered to be the main processes of inflammation ${ }^{9,10}$. Therefore, the treatment of severe NeuA is not limited to corti- 
costeroid use.

Tiotropium bromide (TIO) is a long-acting muscarinic antagonist ${ }^{11}$. It improves asthma by modulating neuronal acetylcholine and decreasing airway smooth muscle thickening $^{12}$. It is usually used as an add-on therapy to the combination of inhaled corticosteroid and long-acting $\beta 2$ agonist in the Global Initiative for Asthma guideline as step 4 or step 5 treatment. TIO inhibits the $\mathrm{M}_{3}$ receptor in the smooth muscle of the airway, which leads to bronchodilation. In an in vitro study, it showed anti-inflammatory effects by controlling proinflammatory cytokine such as interleukin (IL)-8 or IL-17 $\mathrm{A}^{13}$. However, the action of TIO in NeuA remains unclear. This study was conducted to evaluate the effects of TIO in NeuA mouse model.

\section{Materials and Methods}

\section{Mouse experimental model of this study}

We developed a NeuA mouse model in a previous study ${ }^{9}$. Six-week-old female C57BL mice (Orient Bio, Seongnam, Korea) were sensitized with ovalbumin (OVA) (Sigma-Aldrich, St. Louis, MO, USA). OVA (100 $\mu \mathrm{g})$ and aluminum hydroxide (2 mg; Sigma-Aldrich) were dissolved in the saline and injected into the peritoneum to sensitize the $\mathrm{T}_{\mathrm{H}} 2$ inflammation on days 0 and 7 . After sensitization, OVA $(50 \mu \mathrm{g})$ and lipopolysaccharide (LPS) (2 mg/kg) were administered intranasally. Intranasal OVA was administered on days 14, 15, 21, 22, and 23. Intranasal LPS was administered to shift the eosinophilic inflammation to neutrophilic inflammation on days 18, 21, and 23. The mice were sacrificed on days $24^{9,14,15}$ (Figure 1).

\section{Protocol of drug challenge}

The experimental drugs used TIO and dexamethasone
(DEX). TIO (25 $\mu \mathrm{g} / \mathrm{mL})$ was administered by inhalation for 3 minutes per mouse in the inhalation chamber ${ }^{16}$. This was done on days 21, 22, and 23 (Figure 1). DEX (4 mg/kg) was injected into the peritoneum on days 14, 17, 20, and 23 (Figure 1). Mice were randomly allocated and grouped into (1) the control, (2) OVA+LPS $(\mathrm{O}+\mathrm{L})$, (3) OVA+LPS+DEX $(\mathrm{O}+\mathrm{L}+\mathrm{D})$, and (4) OVA+LPS+TIO $(\mathrm{O}+\mathrm{L}+\mathrm{T})$.

\section{Airway resistance}

Airway resistance was measured by the resistance of the respiratory system (Rrs) in the FlexiVent system (SCIREQ, Montreal, QC, Canada). Baseline Rrs in mice was measured by exposure to nebulized phosphate-buffered saline (PBS) for 3 minutes. The Rrs values were re-assessed after exposure to increasing concentrations of methacholine (Sigma-Aldrich) using an Aerosonic ultrasonic nebulizer (DeVilbiss, Somerset, PA, USA). The average values of the Rrs were obtained, which were measured during each 3 minutes sequence for each concentration of methacholine ${ }^{17}$.

\section{Broncho-alveolar lavage}

Broncho-alveolar lavage (BAL) was performed via the cannulated trachea with a silicon tube. We instilled $0.8 \mathrm{~mL}$ of cold sterile PBS into the lung and withdrew the BAL fluid. The BAL fluid was subjected to cytospin for 10 minutes, and then the supernatant was stored at $-80^{\circ} \mathrm{C}$ for further analyses. Fifty microliters aliquots of BAL fluid were centrifuged at $43 \mathrm{~g}$ for 5 minutes and stained with Diff-Quick (Sysmax, Kobe, Japan). Total cell counts were obtained using a hemocytometer LUNA automated cell counter. The percentages of macrophages, eosinophils, lymphocytes, and neutrophils were measured by counting 500 leukocytes in the BAL fluid slides under a light microscope $^{18}$.

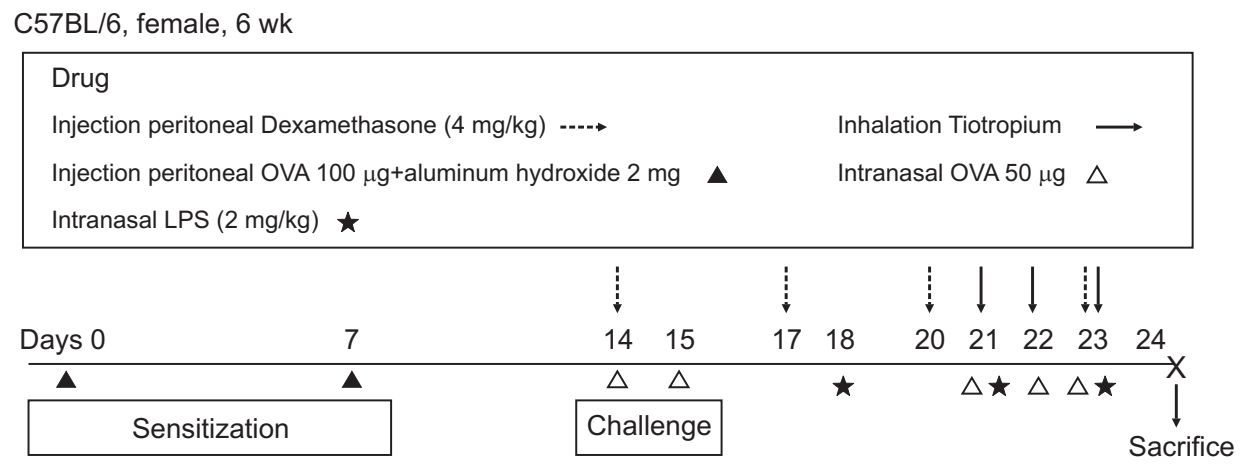

Figure 1. Schematic flow of acute neutrophilic asthma mouse model study. Mice were sensitized with ovalbumin (OVA) on days 0 and 7 . OVA was challenged on days 14, 15, 21, 22, and 23 intranasally. Neutrophilic inflammation was induced by lipopolysaccharides (LPS) which was administered on days 18, 21, and 23 intranasally. Dexamethasone was used for challenge on days 14, 17, 20, and 23 intraperitoneally. Tiotropium bromide was inhaled on days 21, 22, and 23. 


\section{Measurement of inflammatory cytokines}

We measured the levels of inflammatory cytokines in BAL fluid, such as IL-4, IL-5, IL-17A, and IL-22. The levels of IL-1 $\beta$, IL-6, tumor necrosis factor (TNF)- $\alpha$, and interferon (IFN)- $\gamma$ in the lung homogenates were also measured. An enzymelinked immunosorbent assay kits were used for measurement (R\&D Systems, Minneapolis, MN, USA).

\section{Lung tissue histopathology and inflammation score}

The lungs of the mice were fixed in $4 \%$ paraformaldehyde for 24 hours after being sacrificed. The sections were embedded in paraffin and cut into $4 \mu \mathrm{m}$ thickness slides by a microtome. Hematoxylin and eosin (H\&E) staining was performed for microscopic examination. We assessed the inflammation of the peri-bronchial area using a quantitative method that was used in a previous study ${ }^{19}$.

\section{Statistical analyses}

One-way and two-way analysis of variance (ANOVA) were used to evaluate the effect of medication on various measurements in BAL fluid, such as cell counts, total protein, and inflammatory cytokines. The differences in total cell counts and their differences between the medications were compared using repeated-measures ANOVA. Post-hoc analyses using Dunnett's and Turkey's multiple comparison tests were used to compare multiple groups. Statistical significance was set at p-value $<0.05$. All analyses were performed using GraphPad Prism 5.01 (GraphPad Software, San Diego, CA, USA).

\section{Ethics approval}

This study was reviewed and approved by the Institutional Animal Care and Use Committee (IACUC) of the School of Medicine, The Catholic University of Korea (approval no. CUMC-2020-0079-04).

\section{Results}

1. Tiotropium bromide attenuates the airway resistance significantly in neutrophilic asthma

The $\mathrm{O}+\mathrm{L}$ group, which was consistent with the NeuA group, showed a significant increase in airway resistance. DEX, which is the conventional treatment for asthma, did not attenuate the airway resistance of the Rrs in the NeuA mouse model. Unlike DEX, the TIO treated group $(\mathrm{O}+\mathrm{L}+\mathrm{T})$ showed significant improvement in airway resistance compared to the $\mathrm{O}+\mathrm{L}$ group. Interestingly, the $\mathrm{O}+\mathrm{L}+\mathrm{T}$ group showed a similar level of airway resistance compared to that of the control group (Figure 2).

\section{Tiotropium bromide reduces the airway inflammation of neutrophilic asthma}

Neutrophilic inflammation was induced in the $\mathrm{O}+\mathrm{L}$ group compared to that in the control group after OVA and LPS administration. Overall airway inflammation in the BAL fluid was significantly decreased in the $\mathrm{O}+\mathrm{L}+\mathrm{T}$ group. However, no significant changes were observed in the $\mathrm{O}+\mathrm{L}+\mathrm{D}$ group. In particular, neutrophil and eosinophil counts significantly decreased after treatment with TIO. Unlike in the $\mathrm{O}+\mathrm{L}+\mathrm{T}$ group, neutrophils did not decrease after DEX treatment (Figure 3).

Total protein levels were elevated in the $\mathrm{O}+\mathrm{L}$ group. The DEX group did not show significant changes compared with the $\mathrm{O}+\mathrm{L}$ group. However, the total protein levels in the TIO group were significantly improved compared to that in the NeuA group. Inflammation scores by H\&E staining were significant improved after treatment with DEX or TIO. The $\mathrm{O}+\mathrm{L}+\mathrm{T}$ group showed lower inflammation scores than the $\mathrm{O}+\mathrm{L}+\mathrm{D}$ group, but there was no statistically significant difference between the two groups.

\section{Tiotropium bromide has more potent effects than corticosteroids on improving $\mathrm{T}_{\mathrm{H}} 2 / \mathrm{T}_{\mathrm{H}} 17$ inflammation}

After inducing neutrophilic inflammation, IL-5, IL-17A, and

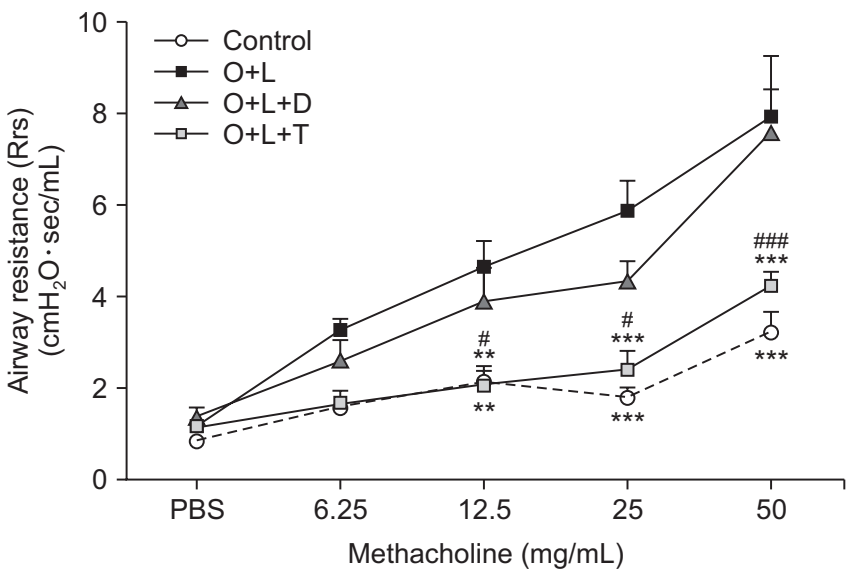

Figure 2. Airway resistance of acute neutrophilic asthma according to medication. Airway resistance is determined by forced oscillation technique. It was measured in response to an increasing dose of methacholine. Tests were proceeded in four groups (control, $\mathrm{O}+\mathrm{L}, \mathrm{O}+\mathrm{L}+\mathrm{D}$, and $\mathrm{O}+\mathrm{L}+\mathrm{T}$ ). The level of airway resistance was elevated in the $\mathrm{O}+\mathrm{L}$ group. However, the $\mathrm{O}+\mathrm{L}+\mathrm{D}$ group did not show improvement of airway hyperresponsiveness. The $\mathrm{O}+\mathrm{L}+\mathrm{T}$ group showed significant attenuated airway resistance compared to the $\mathrm{O}+\mathrm{L}$ group and the $\mathrm{O}+\mathrm{L}+\mathrm{D}$ group. $\mathrm{O}+\mathrm{L}$ vs. all: ${ }^{* *} \mathrm{p}<0.01$, ${ }^{* * *} \mathrm{p}<0.001$; $\mathrm{O}+\mathrm{L}+\mathrm{D}$ vs. $\mathrm{O}+\mathrm{L}+\mathrm{T}:{ }^{*} \mathrm{p}<0.05,{ }^{\# \# \#} \mathrm{p}<0.001$. O: ovalbumin; $\mathrm{L}$ : lipopolysaccharides; D: dexamethasone; T: Tiotropium bromide. 

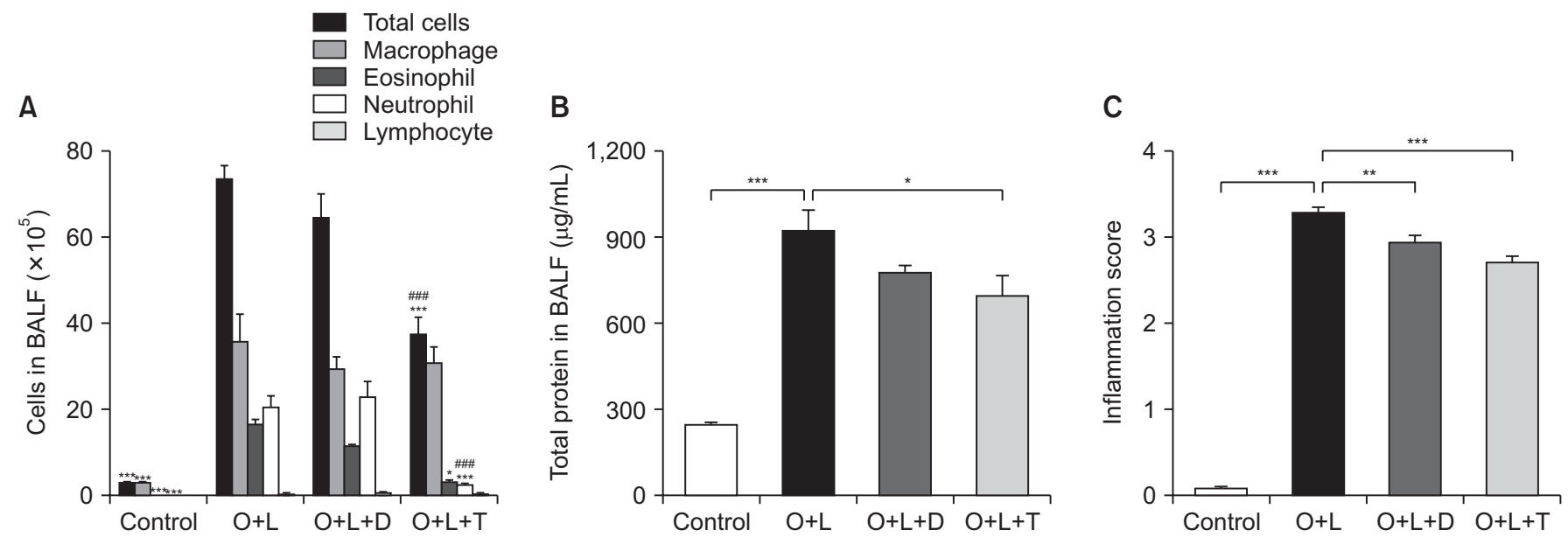

D

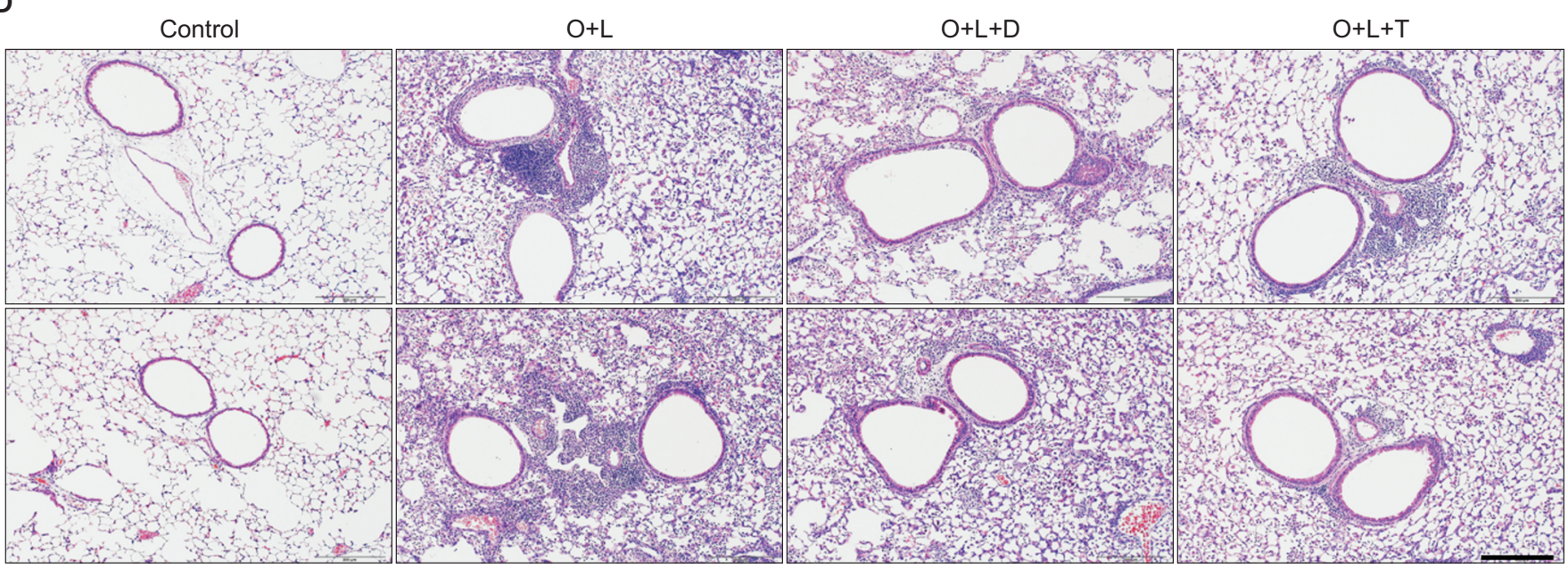

Figure 3. Lung inflammation status of neutrophilic asthma according to the drug used for challenged, such as dexamethasone or Tiotropium bromide. (A, B) Compared to the $\mathrm{O}+\mathrm{L}$ group, the dexamethasone group $(\mathrm{O}+\mathrm{L}+\mathrm{D})$ did not show significant improvement in the level of total cells, neutrophils, eosinophils, or total protein in bronchoalveolar lavage $(\mathrm{BAL})$ fluid. On the contrary, the tiotropium bromide group $(\mathrm{O}+\mathrm{L}+\mathrm{T})$ showed significant improvement of total cells including neutrophils in BAL fluid and total protein level in BAL fluid. $\mathrm{O}+\mathrm{L}$ vs. all: *p<0.05, ${ }^{* *} \mathrm{p}$ $<0.01,{ }^{* * *} \mathrm{p}<0.001 ; \mathrm{O}+\mathrm{L}+\mathrm{D}$ vs. $\mathrm{O}+\mathrm{L}+\mathrm{T}:{ }^{\# \# *} \mathrm{p}<0.001$. (C, D) Inflammation score calculated by hematoxylin and eosin staining of lung tissue histopathology showed improvement both in the $\mathrm{O}+\mathrm{L}+\mathrm{D}$ group and the $\mathrm{O}+\mathrm{L}+\mathrm{T}$ group. O: ovalbumin; L: lipopolysaccharides; $\mathrm{D}$ : dexamethasone; $\mathrm{T}$ : Tiotropium bromide.

IL-22 in the BAL fluid were elevated in the NeuA group compared to the control group. IL-5 and IL-17A levels in the BAL fluid decreased significantly after treatment with TIO, which was not observed after treatment with DEX. IL-22 levels in the BAL fluid showed a similar tendency, but there was no clinical significance (Figure 4). In the analyses of lung homogenates, the levels of IL-1 $\beta$, IL-6, TNF- $\alpha$, and IFN- $\gamma$ were elevated after induction by OVA and LPS. The levels of cytokines in the lung homogenate decreased after being treatment with TIO or with DEX. The TIO group showed more improvement than the DEX group; however, the difference was not statistically significant (Figure 5). TIO showed more potent effects on improving neutrophilic inflammation than DEX. Moreover, $\mathrm{T}_{\mathrm{H}} 2$ and $\mathrm{T}_{\mathrm{H}} 17$-related inflammatory cytokines improved in the TIO group compared to those in the DEX group.

\section{Discussion}

NeuA is characterized by more trapped air, lower lung functions, thicker airway inflammation, and more events of exacerbation than non-NeuA ${ }^{9,20}$. NeuA is often less responsive to the classic asthma medications, such as corticosteroids ${ }^{21-23}$. In a previous study, it was found that $\mathrm{T}_{\mathrm{H}} 17$ and IL-17A play a key role in neutrophilic airway inflammation ${ }^{9}$. Increased neutrophils promote smooth muscle cell proliferation, pro- 
A

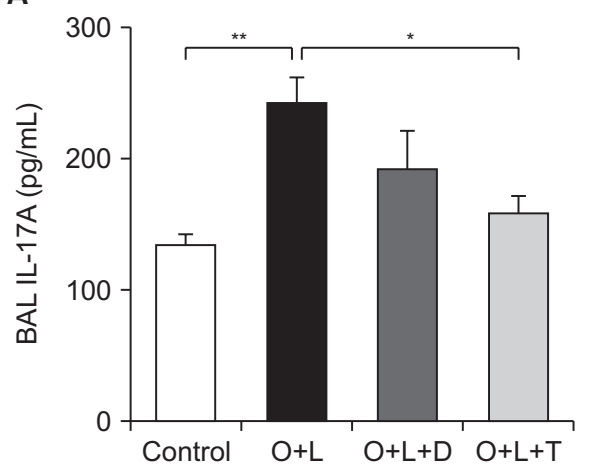

C

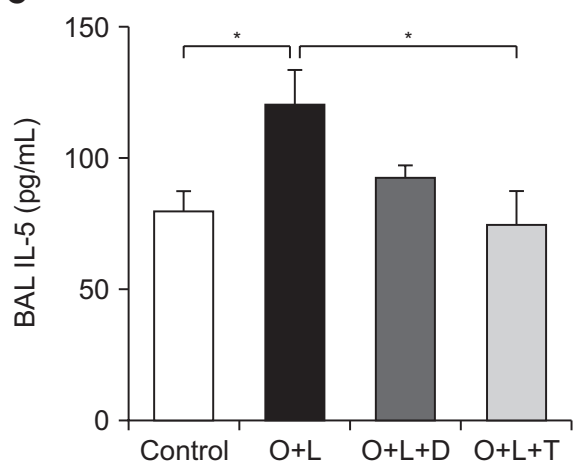

A

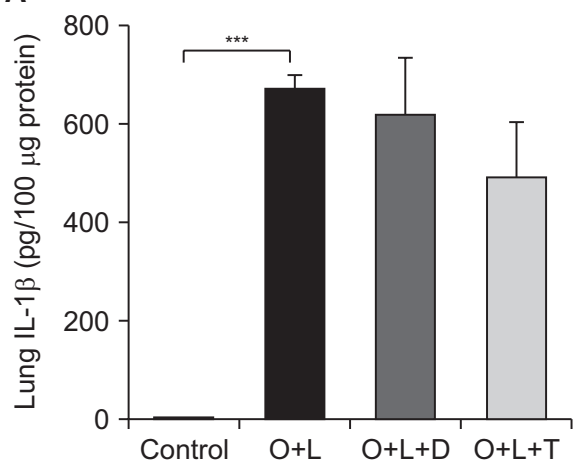

C

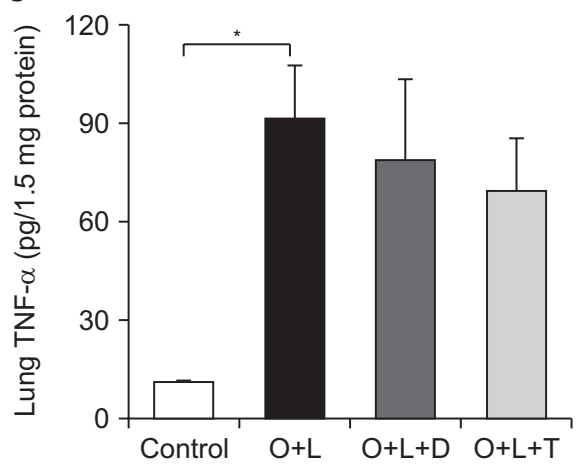

B

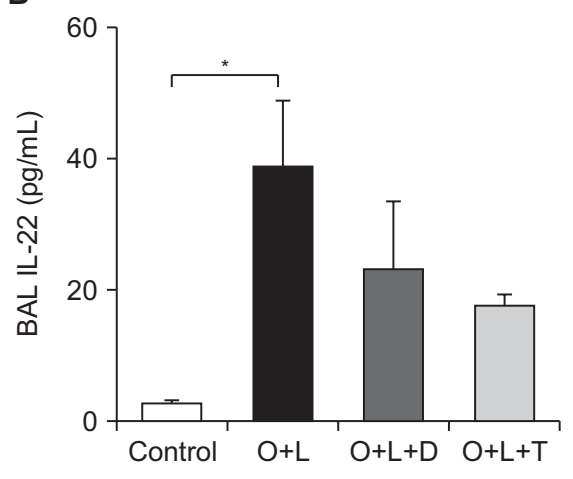

D

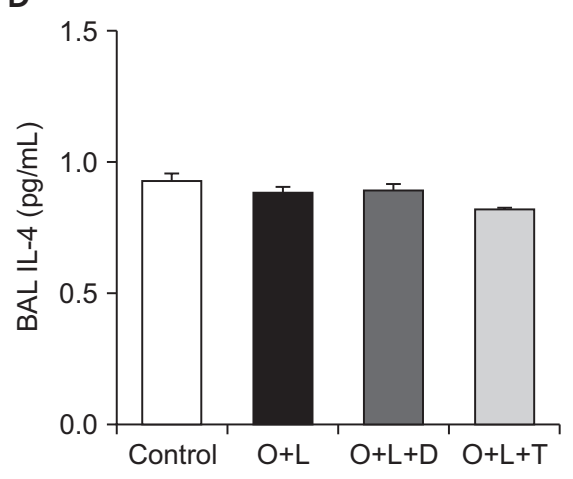

B

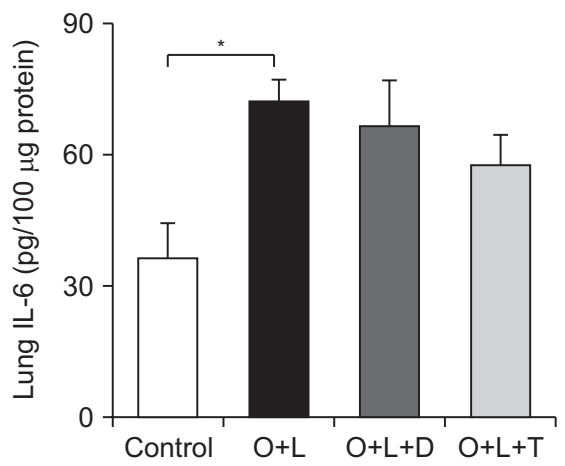

D

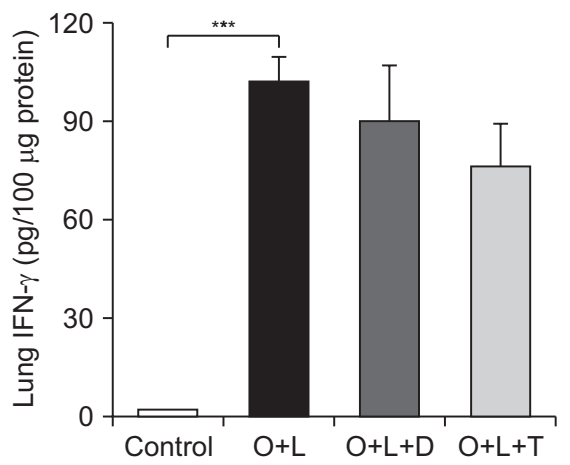

Figure 4. Inflammatory cytokines related to neutrophilic inflammation such as interleukin (IL)-5 and IL-17A in bronchoalveolar lavage (BAL) fluid were attenuated by Tiotropium bromide (A, C). Levels of IL-5 and IL-17A were not significantly attenuated by dexamethasone $(\mathrm{O}+\mathrm{L}+\mathrm{D})$. After dexamethasone and Tiotropium bromide were applied, levels of IL-22 and IL-4 were not differ from those in the neutrophilic asthma group $(\mathrm{O}+\mathrm{L})$ (B, D). O+L vs. all: ${ }^{*} \mathrm{p}<0.05,{ }^{* *} \mathrm{p}<0.01$. O: ovalbumin; L: lipopolysaccharides; D: dexamethasone; T: Tiotropium bromide.

Figure 5. Inflammatory cytokines in lung tissue homogenates showed a declining tendency after treatment with Tiotropium bromide (TIO) without having a clinical significance. T helper cytokines such as interleukin (IL)-1 $\beta$ and IL-6 from lung homogenates (A, B) and pro-inflammatory cytokines such as tumor necrosis factor (TNF)- $\alpha$ and interferon (IFN)- $\gamma$ (C, D) showed a tendency of decline but no clinical significance after treatment with TIO compared to the neutrophilic asthma group. $\mathrm{O}+\mathrm{L}$ vs. all: ${ }^{*} \mathrm{p}<0.05,{ }^{* * *} \mathrm{p}$ $<0.001$. O: ovalbumin; L: lipopolysaccharides; D: dexamethasone; T: Tiotropium bromide. 
inflammatory mediators, and neutrophil recruitment to the airways $^{24}$. These are also possible causes of heterogeneity in airway inflammation, which is difficult to treat.

Many studies have shown that TIO can play potential roles in improving neutrophilic inflammation. TIO improves smooth muscle cell proliferation and remodeling by inhibiting the $\mathrm{M}_{3}$ receptor in the airway. This leads to an improvement in bronchoconstriction. Acetylcholine, which is also controlled by TIO, modulates the airway inflammatory response that is not fully controlled by $\mathrm{T}_{\mathrm{H}} 2$ cells $^{25}$. In contrast to the TIO, there are several reasons that corticosteroid is not effective for controlling the neutrophilic inflammation in the NeuA. NeuA is mainly mediated by $\mathrm{T}_{\mathrm{H}} 17$ cells, which are not controlled by corticosteroids. In a previous study, NeuA was shown to have corticosteroid-insensitivity, which was mediated by histone deacetylase 2 inactivity ${ }^{9}$. Therefore, we conducted this study to evaluate the effect of TIO alone on NeuA. We revealed some aspects of TIO as an appropriate drug for controlling NeuA.

First, TIO showed a significant improvement in the inflammation in NeuA. Neutrophil counts significantly improved after TIO treatment. Total protein, IL-17A, and IL-5 in the BAL fluid, which were related to $T_{H} 1$ and $T_{H} 17$ responses, were decreased after TIO treatment in the NeuA group. In this study, we demonstrated the effect of TIO on neutrophilic inflammation by controlling $\mathrm{T}_{\mathrm{H}} 1$ and $\mathrm{T}_{\mathrm{H}} 17$ cells. Second, TIO showed more improvement in inflammatory cytokines than DEX. TIO showed clinically relevant improvement compared to that of DEX, which did not show any statistically significant difference. Third, airway resistance after TIO treatment was attenuated in the control group. This means that the TIO showed improved the airway flow in NeuA which is the main target of asthma care. Based on these results, we can assume the possibility of early administration of TIO in NeuA. Fourth, we administrated TIO via inhalation method, which was the proper way of delivery as originally designed. In many previous studies, TIO was usually administered by intratracheal or intraperitoneal method, which is not the right way of using TIO. Therefore, we developed a proper TIO inhalation mouse model for further evaluation.

There were a few limitations to this study. First, we did not observe a dose-dependent relationship between TIO and NeuA. However, the decided level of TIO treatment was calculated using a minimal animal equivalent dose of human drug. Therefore, we showed minimal effects of TIO in the NeuA mouse model. Second, we did not discover a possible pathway for controlling neutrophilic inflammation using TIO. We simply showed the effect of TIO on NeuA. We are planning to conduct an upcoming study to evaluate the possible mechanism of TIO.

In this study, we demonstrated the therapeutic effects of TIO in NeuA. These results show the possibility of TIO as the primary medication in NeuA. Further studies on the $T_{H} 1 / T_{H} 17$ inflammation cascade of TIO should be conducted in the future.

\section{Authors' Contributions}

Conceptualization: Yoon HK. Methodology: An TJ, Park CK, Yoon HK. Data curation: Kim JH. Formal analysis: Kim JH. Investigation: Park CK, Yoon HK. Validation: An TJ, Yoon HK. Writing - original draft: An TJ. Writing - review and editing: An TJ. Approval of final manuscript: all authors.

\section{Conflicts of Interest}

No potential conflict of interest relevant to this article was reported.

\section{Funding}

This Study was supported by a 2019-Grant from The Korean Academy of Tuberculosis and Respiratory Diseases.

\section{References}

1. Pembrey L, Barreto ML, Douwes J, Cooper P, Henderson J, Mpairwe H, et al. Understanding asthma phenotypes: the World Asthma Phenotypes (WASP) international collaboration. ERJ Open Res 2018;4:00013-2018.

2. Rhee CK, Kang JY, Park CK, Lee SY, Kwon SS, Kim YK, et al. Effect of nilotinib on airway remodeling in a murine model of chronic asthma. Exp Lung Res 2014;40:199-210.

3. FitzGerald JM, Tran TN, Alacqua M, Altraja A, Backer V, Bjermer L, et al. International severe asthma registry (ISAR): protocol for a global registry. BMC Med Res Methodol 2020;20:212.

4. ISAR Study Group. International Severe Asthma Registry: Mission Statement. Chest 2020;157:805-14.

5. King GG, James A, Harkness L, Wark PA. Pathophysiology of severe asthma: We've only just started. Respirology 2018;23:262-71.

6. Seys SF, Lokwani R, Simpson JL, Bullens DM. New insights in neutrophilic asthma. Curr Opin Pulm Med 2019;25:113-20.

7. Allam V, Sukkar MB. Investigating MIF in mouse models of severe corticosteroid-resistant neutrophilic asthma. Methods Mol Biol 2020;2080:203-12.

8. Zhang X, Xie J, Sun H, Wei Q, Nong G. sRAGE inhibits the mucus hypersecretion in a mouse model with neutrophilic asthma. Immunol Invest 2021 May 21 [Epub]. https://doi.org/ 10.1080/08820139.2021.1928183.

9. An TJ, Rhee CK, Kim JH, Lee YR, Chon JY, Park CK, et al. Effects of macrolide and corticosteroid in neutrophilic asthma mouse model. Tuberc Respir Dis (Seoul) 2018;81:80-7.

10. Zhang X, Zhang M, Jiang M, Nong G. Effect of IL7 on Th17 cell responses in a mouse model of neutrophilic asthma. Mol 
Med Rep 2020;22:1205-12.

11. Radovanovic D, Santus P, Blasi F, Mantero M. The evidence on tiotropium bromide in asthma: from the rationale to the bedside. Multidiscip Respir Med 2017;12:12.

12. Hamelmann E, Vogelberg C, Szefler SJ. Tiotropium for the treatment of asthma in adolescents. Expert Opin Pharmacother 2017;18:305-12.

13. Anzalone G, Gagliardo R, Bucchieri F, Albano GD, Siena L, Montalbano AM, et al. IL-17A induces chromatin remodeling promoting IL-8 release in bronchial epithelial cells: effect of Tiotropium. Life Sci 2016;152:107-16.

14. Zhao S, Jiang Y, Yang X, Guo D, Wang Y, Wang J, et al. Lipopolysaccharides promote a shift from Th2-derived airway eosinophilic inflammation to Th17-derived neutrophilic inflammation in an ovalbumin-sensitized murine asthma model. J Asthma 2017;54:447-55.

15. Bergquist M, Jonasson S, Hjoberg J, Hedenstierna G, Hanrieder J. Comprehensive multiplexed protein quantitation delineates eosinophilic and neutrophilic experimental asthma. BMC Pulm Med 2014;14:110.

16. Zhao S, Yang Q, Yu Z, Lv Y, Zhi J, Gustin P, et al. Protective effects of tiotropium alone or combined with budesonide against cadmium inhalation induced acute neutrophilic pulmonary inflammation in rats. PLoS One 2018;13:e0193610.

17. McGovern TK, Robichaud A, Fereydoonzad L, Schuessler TF, Martin JG. Evaluation of respiratory system mechan- ics in mice using the forced oscillation technique. J Vis Exp 2013:e50172.

18. Lee HY, Rhee CK, Kang JY, Park CK, Lee SY, Kwon SS, et al. Effect of intranasal rosiglitazone on airway inflammation and remodeling in a murine model of chronic asthma. Korean J Intern Med 2016;31:89-97.

19. Myou S, Leff AR, Myo S, Boetticher E, Tong J, Meliton AY, et al Blockade of inflammation and airway hyperresponsiveness in immune-sensitized mice by dominant-negative phosphoinositide 3-kinase-TAT. J Exp Med 2003;198:1573-82.

20. Ray A, Kolls JK. Neutrophilic inflammation in asthma and association with disease severity. Trends Immunol 2017;38:94254

21. Park SW, Park JS, Jeong SH, Lee YN, Hwangbo Y, Park JS, et al. Air trapping is a major determinant of persistent airway obstruction in asthmatics. Respir Med 2012;106:786-93.

22. Kalchiem-Dekel O, Yao X, Levine SJ. Meeting the challenge of identifying new treatments for type 2-low neutrophilic asthma. Chest 2020;157:26-33.

23. Gibson PG, Foster PS. Neutrophilic asthma: welcome back! Eur Respir J 2019;54:1901846.

24. Crisford H, Sapey E, Rogers GB, Taylor S, Nagakumar P, Lokwani R, et al. Neutrophils in asthma: the good, the bad and the bacteria. Thorax 2021;76:835-44.

25. Gosens R, Gross N. The mode of action of anticholinergics in asthma. Eur Respir J 2018;52:1701247. 